\title{
Ichtyofaune de la rivière Dodo (Côte d'ivoire, Afrique de l'ouest) : mise à jour et influence des variables environnementales sur la distribution des espèces
}

\author{
Tanoh Marius KAMELAN, Stanislas Silvain YAO*, Kouakou Augustin KOUAMÉ, Konan Gervais N'ZI, \\ Essetchi Paul KOUAMÉLAN \\ Laboratoire d'Hydrobiologie, UFR Biosciences, Université Félix Houphouët-Boigny, 22 BP 582 Abidjan 22, Côte d'Ivoire. \\ *Auteur correspondant, E-mail : yaosilvain@yahoo.com Tél : +225 07641600 / 46256781
}

Original submitted in on $12^{\text {th }}$ July 2013 Published online at www.m.elewa.org on $30^{\text {th }}$ November 2013. https://dx.doi.org/10.4314/jab.v71i1.98824

\section{RÉSUMÉ}

Objectifs : Cette étude vise à mettre à jour l'inventaire de l'ichtyofaune de la rivière Dodo, à analyser la distribution des espèces et à préciser les variables environnementales qui influencent cette distribution.

Méthodologie et Résultats : Les poissons ont été échantillonnés entre Juillet 2008 et Avril 2009. Au total 39 espèces reparties entre 30 genres et 20 familles ont été identifiées. Vingt-trois (23) espèces sont signalées pour la première fois dans la rivière Dodo, tandis que dix espèces préalablement connues de ce bassin n'ont pas été capturées. Les espèces majoritaires sont Gobionellus occidentalis et Hemichromis fasciatus. Les espèces les plus largement distribuées sont Chromidotilapia guntheri et Hemichromis fasciatus. La distribution des espèces est influencée par le taux d'oxygène dissous, la largeur du bassin versant, la nature du substrat, les plantes aquatiques et la canopée.

Conclusion et application : Cette étude a permis d'actualiser l'inventaire de l'ichtyofaune de la rivière Dodo et d'identifier pour la première fois les paramètres environnementaux qui influencent la distribution des espèces. Face à l'anthropisation progressive des berges et du bassin de la Dodo, ces résultats serviront de références pour les investigations ultérieures de suivi de l'état de la faune ichtyologique et à l'élaboration de mesures adéquates pour éviter la perte de la biodiversité aquatique.

Mots clés : rivière Dodo, ichtyofaune, inventaire, distribution, variables environnementales

Ichthyofauna of Dodo River (Ivory Coast, West Africa): Update of inventory, distribution and influence of environmental parameters

\section{ABSTRACT}

Objectives: This study aims to update the inventory of ichtyofauna of Dodo River, analyze the distribution of fishes and indicate the environmental variables, which influence this distribution.

Methodology and Results: The ichthyofauna of Dodo River was collected from July 2008 to April 2009. Thirtynine (39) species belonging to 30 genus and 20 families were recorded. Twenty-three (23) species were reported for the first time from the Dodo River, whereas, 10 species previously known from this river have not been recorded. The most abundant species were Gobionellus occidentalis and Hemichromis fasciatus. The 
species Chromidotilaipia guntheri and Hemichromis fasciatus had the highest frequencies of occurrence. The main environmental factors influencing fish distribution were dissolved oxygen, river width, substrate types, aquatic plants and canopy closure.

Conclusion and application: This study allowed to update fish biodiversity of the Dodo River and to identify for the first time environmental factors which influence the distribution of fish species. With the increase of human activities in bank of Dodo River and in its basin, these results will be references to follow through condition of ichthyofauna and to take appropriate steps to avoid losses in aquatic biodiversity

Key words: Dodo River, ichthyofauna, inventory, distribution, environmental variables

\section{INTRODUCTION}

Les écosystèmes aquatiques fournissent beaucoup de biens et de services qui ne sont pas souvent appréciés à leur juste valeur (Brummett et al., 2008). L'ichtyofaune, qui est l'ensemble des poissons d'un cours d'eau, constitue en particulier une source de protéines à coût réduit et de revenus pour les populations riveraines. Malheureusement, les activités anthropiques deviennent de plus en plus menaçantes pour les poissons (Yao, 2006). En effet, les conséquences des captures intensives, l'utilisation des substances chimiques en agriculture, la construction de barrages hydroélectriques et de barrages d'irrigation, la destruction des forêts et les introductions de nouvelles espèces de poisson perturbent fortement les écosystèmes aquatiques et font planer de réelles menaces de disparition sur de nombreuses espèces de poisson (Kamdem \& Teugels, 1998 ; Gourène et al., 1999). En Afrique de l'ouest, la construction de barrages, le déboisement intensif et l'utilisation de produits chimiques en agriculture demeurent encore d'actualité. La question de l'avenir de la faune ichtyologique devient alors une préoccupation majeure.

La rivière Dodo est l'un des petits bassins côtiers situés dans les derniers massifs forestiers du sudouest de la Côte d'Ivoire. A l'instar de nombreux hydrosystèmes africains (Lévêque \& Paugy, 2006a), ce cours d'eau est sous l'influence de plusieurs activités anthropiques car l'ensemble du couvert forestier du bassin versant de la rivière a été transformé en zone agricole avec l'implantation de blocs agro-industriels d'hévéa, de palmiers à huile, de caféiers et de cacaoyers. De plus, des canalisations sont effectuées sur son lit principal pour l'irrigation des plantations industrielles de la société de caoutchouc de Grand-Bereby (SOGB). Par ailleurs, des sites de toilettes et de lessive y sont aménagés par les riverains. Ces pressions anthropiques, si rien n'ait fait, pourraient à long terme perturber l'ensemble de l'environnement aquatique et plus particulièrement l'ichtyofaune. Des mesures vigoureuses de restauration et de conservation doivent alors être prises et exécutées. Cependant, cette politique de préservation requiert au préalable une bonne connaissance des espèces de poissons et la caractérisation des facteurs qui influencent leur distribution (Lalèyè et al., 2004 ; Wu et al., 2011).

C'est dans cette optique que la présente étude a été conduite sur la rivière Dodo. Aussi, très peu d'informations existent-il sur ce bassin côtier en général et sur son ichtyofaune en particulier. Les listes des espèces de poissons disponibles pour ce cours d'eau remontent à deux décennies (Teugels et al., 1988 ; Paugy et al., 1994). Elles indiquent respectivement que 21 et 25 espèces sont présentes dans cette rivière. Cependant, il n'existe aucune donnée ni sur l'abondance relative des espèces, ni sur leur répartition le long du bassin, encore moins sur les facteurs qui déterminent cette distribution.

En outre, la présente étude s'inscrit dans le cadre d'un vaste programme d'inventaire et/ou de mise à jour de l'inventaire des bassins côtiers ivoiriens. Elle vise donc à: (1) mettre à jour l'inventaire ichtyologique de la rivière Dodo, (2) analyser la distribution des espèces et (3) déterminer les variables environnementales qui régissent cette distribution. 


\section{MATÉRIEL ET MÉTHODES}

Milieu d'étude: Localisée au sud-ouest de la Côte d'Ivoire, la rivière Dodo prend sa source dans la forêt classée de la Haute Dodo et se jette dans l'océan Atlantique au niveau de Mami-Bereby (Figure 1). Elle a

une longueur de $56 \mathrm{~km}$ pour un bassin versant de 850 $\mathrm{km}^{2}$ (Teugels et al., 1988). Trente-cinq sites d'échantillonnage (D1 à D35) ont été retenus sur l'ensemble du bassin en fonction de leur accessibilité.

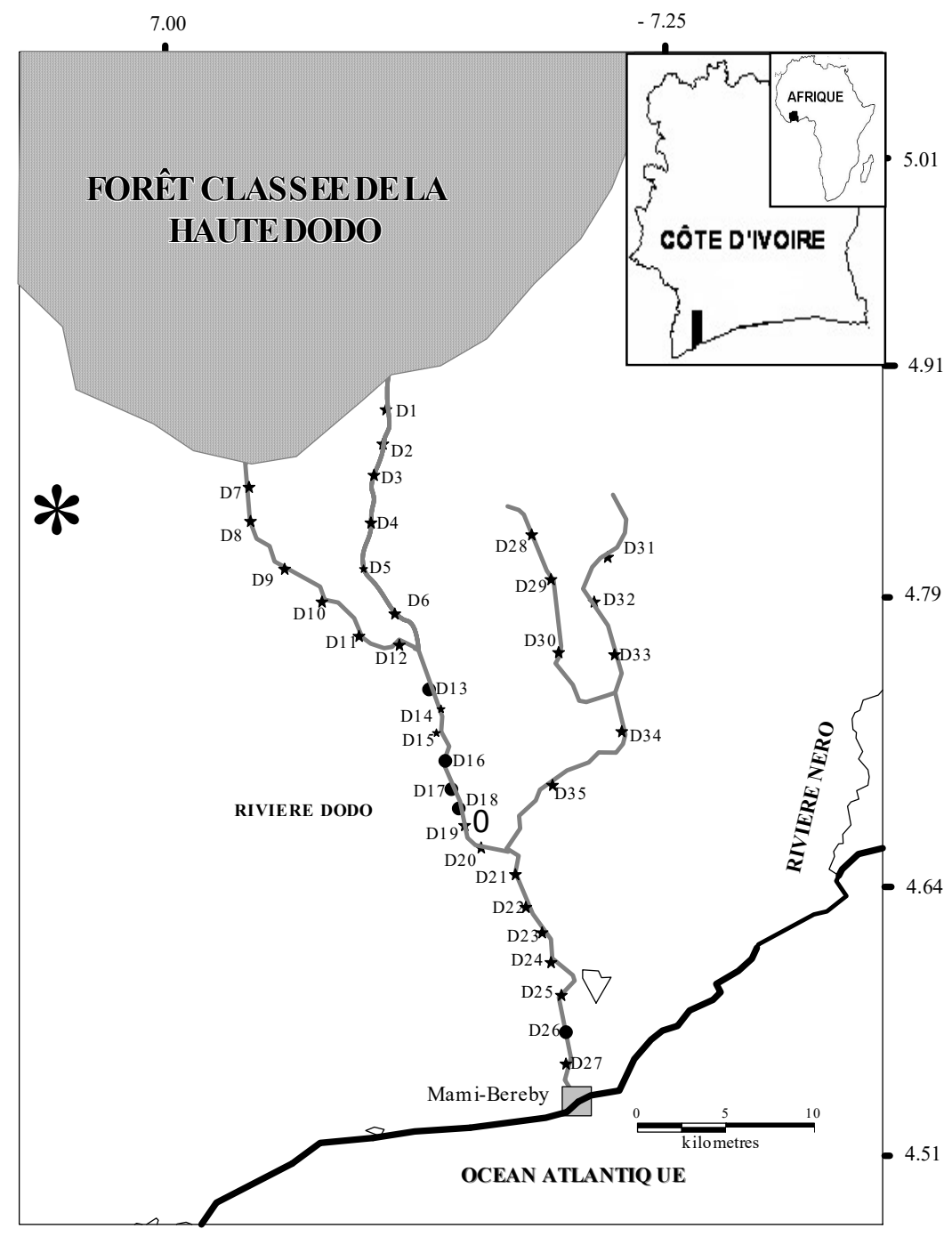

Figure 1: Situation géographique de la rivière Dodo et localisation des stations d'échantillonnage. $\bullet$ = filet maillant ; $\star$ = pêche électrique.

Échantillonnage des poissons : Les poissons ont été capturés en juillet et octobre 2008, puis en janvier et avril 2009. Une batterie de 17 filets monofilaments de vide de mailles $8,10,12,15,20,25,30,35,40,45,50,55,60$, $65,70,80$ et $90 \mathrm{~mm}$ et un appareil de pêche électrique de marque Smith Root Backpack modèle 12-B Pow ont servi à l'échantillonnage. Chaque filet maillant mesure $30 \mathrm{~m}$ de long et $1,5 \mathrm{~m}$ de hauteur de chute. Les filets maillants ont été utilisés dans les stations D13, D16, D17, D18 et D26. Ils ont été posés à $17 \mathrm{~h}$ et visités à $7 \mathrm{~h}$ le lendemain pour la pêche nocturne, puis visités de nouveau et relevés à $12 \mathrm{~h}$ pour la pêche diurne. Les pêches électriques se sont déroulées dans les stations D1 à D12, D14, D15, D19 à D25 et D27 à D35. Les poissons capturés ont été identifiés selon Paugy et al. (2003a, b). 
Mesure des variables environnementales : Sur chaque site, avant chaque pêche expérimentale, les paramètres physico-chimiques suivants ont été mesurés: la profondeur et la largeur du cours d'eau (mesurées en $\mathrm{m}$ ), la vitesse du courant (mesurée en $\mathrm{m} / \mathrm{s}$ avec un courantomètre de type Valeport 008), le pH (mesuré avec un pH-mètre WTW-pH 330), le taux d'oxygène dissous (mesuré en mg/l à l'aide d'un oxymètre WTW DIGI 330), la conductivité et le taux de solides dissous ou TDS (respectivement en $\mu \mathrm{S} / \mathrm{cm}$ et $\mathrm{mg} / \mathrm{l}$ avec un conductimètre WTW-LF 340), la température (en ${ }^{\circ} \mathrm{C}$ avec un thermomètre couplé à l'oxymètre) et la transparence (mesurée en cm à l'aide d'un disque de Secchi). Les taux (en \%) de fermeture de la canopée, de couverture des eaux par les plantes aquatiques, et de substrat (sable, mélange sable-gravier, gravier, boue, rocher, mélange argile-boue, mélange bois mort-feuilles-racines) ont été aussi notés.

Traitements des données: Compte tenu du nombre relativement faible de stations échantillonnées aux filets maillants ( $n=5$ sur 35 stations) et hormis l'inventaire qualitatif et la zonation longitudinale, seuls les spécimens récoltés à la pêche électrique ont été considérés pour les différentes analyses.

Calcul des pourcentages d'occurrence ou fréquences $F$ des espèces: Le pourcentage d'occurrence ou fréquence $F(\%)$ est le pourcentage de stations où une espèce i a été échantillonnée par rapport au nombre total de stations prospectées (Gbenyedji et al.,

\section{RÉSULTATS}

Inventaire qualitatif de l'ichtyofaune : Les spécimens de poissons capturés au cours de l'ensemble des campagnes d'échantillonnage se répartissent entre 9 ordres, 20 familles, 30 genres et 39 espèces (Tableau 1). Cette composition du peuplement ichtyologique comprend 31 espèces dulçaquicoles, 7 espèces à affinité estuarienne et/ou marine (Dalophis boulengeri, Pellonula leonensis, Sarotherodon melanotheron, Tilapia guineensis, Liza falcipinnis, Awaous lateristriga et Gobionellus occidentalis) et une espèce hybride (Tilapia guineensis x Tilapia zillii). L'ordre le plus riche est celui
2011). Elle quantifie le degré d'ubiquité des différentes espèces :

$\mathrm{F}=\frac{\mathrm{Si}}{\mathrm{St}} \times 100$

Où : $\mathrm{Si}=$ nombre de stations où l'espèce $\mathrm{i}$ a été capturée ; St = nombre total de stations prospectées. La classification des espèces en fonction de la fréquence $F(\%)$ s'établit comme suit :

$F<10$ : espèce très rare; $10 \leq F<20$ : espèce accidentelle ou rare ; $20 \leq F<40$ : espèce accessoire ; $40 \leq F<60$ : espèce assez fréquente ; $60 \leq F<80$ : espèce fréquente; $80 \leq F$ : espèce très fréquente.

Zonation longitudinale de la rivière: En l'absence d'éléments physiques (barrage, chute d'eau, présence d'écosystème particulier), la méthode de N'Douba et al. (2003) a été utilisée pour différencier les différentes sections de la rivière. Selon ces auteurs, la limite supérieure du cours inférieur est la station la plus éloignée de l'océan Atlantique où est capturée une espèce de poisson à affinité marine et/ou estuarienne.

Étude de l'influence des variables environnementales: Les principales variables environnementales qui influencent la distribution des poissons ont été mises en évidence par une analyse canonique de correspondance (ACC) (Ter Braak et Šmilauer, 2002). L'ACC a été couplée à des tests de Monte Carlo qui permettent de sélectionner les variables environnementales qui expliquent le mieux la distribution des espèces de poissons.

des Perciformes qui regroupe 6 familles et 15 espèces. II est suivi de celui des Siluriformes avec 4 familles et 7 espèces. Les Anguilliformes et les Clupéiformes présentent moins de taxon avec une famille et une espèce. La famille des Cichlidae renferme plus d'espèces $(n=8)$. Elle est suivie par la famille des Clariidae $(n=4)$, ensuite de celle des Alestidae, Cyprinidae et Gobiidae avec 3 espèces chacune, puis des Mormyridae, des Syngnathidae et des Nothobranchiidae avec 2 espèces chacune. Toutes les autres familles sont monospécifiques. 
Tableau 1 : Liste, occurrence et distribution des espèces de poissons échantillonnées dans la rivière Dodo en Juillet et Octobre 2008, et en Janvier et Avril 2009.

\begin{tabular}{|c|c|c|c|c|c|}
\hline Ordres et familles & Espèces & Codes & $\begin{array}{c}\text { Nombre de } \\
\text { stations } \\
\text { (total } n= \\
30) \\
\end{array}$ & $\begin{array}{c}\text { Pourcentage } \\
\text { d'occurrence } \\
(\%)\end{array}$ & $\begin{array}{l}\text { Distribution et } \\
\text { occurrence }\end{array}$ \\
\hline $\begin{array}{l}\text { Anguilliformes } \\
\text { Ophichthidae* }\end{array}$ & Dalophis boulengerił* & D bou & - & 6,67 & $\operatorname{lnf} ; \mathrm{Tr}$ \\
\hline $\begin{array}{l}\text { Clupeiformes* } \\
\text { Clupeidae* }^{*}\end{array}$ & Pellonula leonensis ${ }^{*}$ & \multicolumn{4}{|c|}{ Capturée au filet maillant } \\
\hline $\begin{array}{l}\text { Osteoglossiformes }^{*} \\
\text { Notopteridae*}^{*} \\
\text { Mormyridae* }^{*}\end{array}$ & $\begin{array}{l}\text { Papyrocranus afer* } \\
\text { Marcusenius ussheri* } \\
\text { Petrocephalus bovei* }\end{array}$ & \multicolumn{4}{|c|}{$\begin{array}{l}\text { Capturée au filet maillant } \\
\text { Capturée au filet maillant }\end{array}$} \\
\hline $\begin{array}{l}\text { Characiformes } \\
\text { Hepsetidae } \\
\text { Alestidae }\end{array}$ & $\begin{array}{l}\text { Hepsetus odoe } \\
\text { Brycinus longipinnis } \\
\text { Brycinus macrolepidotus* } \\
\text { Micralestes occidentalis* }\end{array}$ & \multicolumn{4}{|c|}{$\begin{array}{l}\text { Capturée au filet maillant } \\
12 \quad 40 \\
\text { Capturée au filet maillant } \\
\text { Capturée au filet maillant }\end{array}$} \\
\hline $\begin{array}{l}\text { Cypriniformes } \\
\text { Cyprinidae }\end{array}$ & $\begin{array}{l}\text { Barbus ablabes } \\
\text { Barbus trispilos } \\
\text { Barbus wurtzi }\end{array}$ & $\begin{array}{l}\text { B abl } \\
\text { B tri }\end{array}$ & $\begin{array}{c}7 \\
7 \\
\text { Capturé }\end{array}$ & $\begin{array}{c}23,33 \\
23,33 \\
\text { e au filet maillant }\end{array}$ & $\begin{array}{l}\text { Inf ; Sup ; As } \\
\text { Inf ; Sup ; As }\end{array}$ \\
\hline $\begin{array}{l}\text { Siluriformes } \\
\text { Claroteidae* }^{*} \\
\text { Schilbeidae* } \\
\text { Amphiliidae } \\
\text { Clariidae }\end{array}$ & $\begin{array}{l}\text { Chrysichthys nigrodigitatus* } \\
\text { Schilbe mandibularis* } \\
\text { Amphilius atesuensis* } \\
\text { Clarias buettikoferi* } \\
\text { Clarias ebriensis } \\
\text { Heterobranchus isopterus* } \\
\text { Heterobranchus longifilis* }\end{array}$ & $\begin{array}{l}\mathrm{C} \text { nig } \\
\mathrm{A} \text { ate } \\
\mathrm{C} \text { bue } \\
\mathrm{H} \text { iso } \\
\mathrm{H} \text { lon }\end{array}$ & $\begin{array}{l}1 \\
\text { Capturé } \\
3 \\
4 \\
\text { Capturé } \\
9 \\
1 \\
\end{array}$ & $\begin{array}{c}3,33 \\
\text { e au filet maillant } \\
10 \\
13,33 \\
\text { e au filet maillant } \\
30 \\
3,33 \\
\end{array}$ & $\begin{array}{c}\text { Sup ; Tr } \\
\text { Sup ; Ac } \\
\text { Inf ; Sup ; Ac } \\
\text { Inf ; Sup ; As } \\
\text { Sup ; Tr }\end{array}$ \\
\hline $\begin{array}{l}\text { Syngnathiformes* } \\
\text { Syngnathidae* }^{*}\end{array}$ & $\begin{array}{l}\text { Enneacampus kaupi* } \\
\text { Microphis brachyurus } \\
\text { aculeatus* }\end{array}$ & $\begin{array}{c}\text { E kau } \\
\text { M b acu }\end{array}$ & $\begin{array}{l}4 \\
1\end{array}$ & $\begin{array}{c}13,33 \\
3,33\end{array}$ & $\begin{array}{l}\text { Sup ; Ac } \\
\text { Inf ; Tr }\end{array}$ \\
\hline $\begin{array}{l}\text { Cyprinodontiformes } \\
\text { Poeciliidae } \\
\text { Nothobranchiidae }\end{array}$ & $\begin{array}{l}\text { Aplocheilichthys schioetzi* } \\
\text { Epiplatys chaperi } \\
\text { Epiplatys dageti }\end{array}$ & $\begin{array}{l}\text { A sch } \\
\text { E cha } \\
\text { E dag }\end{array}$ & $\begin{array}{c}9 \\
1 \\
12 \\
\end{array}$ & $\begin{array}{c}30 \\
3,33 \\
40 \\
\end{array}$ & $\begin{array}{l}\text { Inf ; Sup ; As } \\
\text { Inf ; Tr } \\
\text { Inf ; Sup ; Af }\end{array}$ \\
\hline $\begin{array}{l}\text { Perciformes } \\
\text { Channidae } \\
\text { Cichlidae }\end{array}$ & $\begin{array}{l}\text { Parachanna obscura } \\
\text { Chromidotilapia guntheri } \\
\text { Hemichromis fasciatus } \\
\text { Sarotherodon melanotheron }{ }^{1} \\
\text { Tilapia guineensis }{ }^{1} \\
\text { Tilapia guineensis x T. zillii2* }^{*} \\
\text { Tilapia mariae } \\
\text { Tilapia zillii* }\end{array}$ & $\begin{array}{l}\text { P obs } \\
\text { C gun } \\
H \text { fas } \\
\text { S mel } \\
\text { T gui } \\
\text { T hyb } \\
\text { T mar } \\
\text { T zil }\end{array}$ & $\begin{array}{l}7 \\
22 \\
20 \\
2 \\
2 \\
4 \\
2 \\
1\end{array}$ & $\begin{array}{c}23,33 \\
73,33 \\
66,67 \\
6,67 \\
6,67 \\
13,33 \\
6,67 \\
3,33\end{array}$ & $\begin{array}{l}\text { Inf ; Sup ; As } \\
\text { Inf ; Sup ; F } \\
\text { Inf ; Sup ; F } \\
\text { Sup ; Tr } \\
\text { Inf ; Sup ; Tr } \\
\text { Inf } \text { Sup ; Ac } \\
\text { Sup ; Tr } \\
\text { Sup ; Tr }\end{array}$ \\
\hline
\end{tabular}




\begin{tabular}{|c|c|c|c|c|c|}
\hline & Thysochromis ansorgii* & T ans & 1 & 3,33 & Sup ; Tr \\
\hline Mugilidae* & Liza falcipinnis ${ }^{4 *}$ & \multicolumn{4}{|c|}{ Capturée au filet maillant } \\
\hline \multirow[t]{3}{*}{ Gobiidae } & Awaous lateristriga ${ }^{1}$ & A lat & 3 & 10 & Inf ; Sup ; Ac \\
\hline & Gobionellus occidentalis ${ }^{1 *}$ & G occ & 1 & 3,33 & $\operatorname{lnf} ; \mathrm{Tr}$ \\
\hline & Parasicydium bandama & $\mathrm{P}$ ban & 3 & 10 & Inf ; Sup ; Ac \\
\hline Eleotridae* & Kribia nana* & Knan & 1 & 3,33 & Sup ; Tr \\
\hline Anabantidae & Ctenopoma petherici & C pet & 4 & 13,33 & Inf ; Sup ; Ac \\
\hline
\end{tabular}

Inventaire quantitatif de l'ichtyofaune : Au niveau des ordres, les Perciformes constituent plus de la moitié des captures à $68,52 \%$ (Figure 2). Ils sont suivis des Cypriniformes $(9,56 \%)$, Cyprinodontiformes $(8,43 \%)$, Siluriformes $(7,53 \%)$ et des Characiformes $(4,82 \%)$. Les autres ordres (Syngnathiformes, Osteoglossiformes et Anguilliformes) moins représentés, constituent $1,13 \%$ des échantillons. Les familles les plus abondantes sont celles des Cichlidae (39,83\%) et des Gobiidae (27,03\%) (Figure 3). Viennent ensuite les Cyprinidae (9,56\%), Clariidae $(7,00 \%)$ et les Nothobranchiidae $(6,02 \%)$. Les dix autres familles représentent $10,54 \%$ des effectifs. Les espèces majoritaires sont Gobionellus occidentalis $(25,68 \%)$ et Hemichromis fasciatus (23,87\%) (Figure 4). Chacune des autres espèces représente moins de $10 \%$ des effectifs.

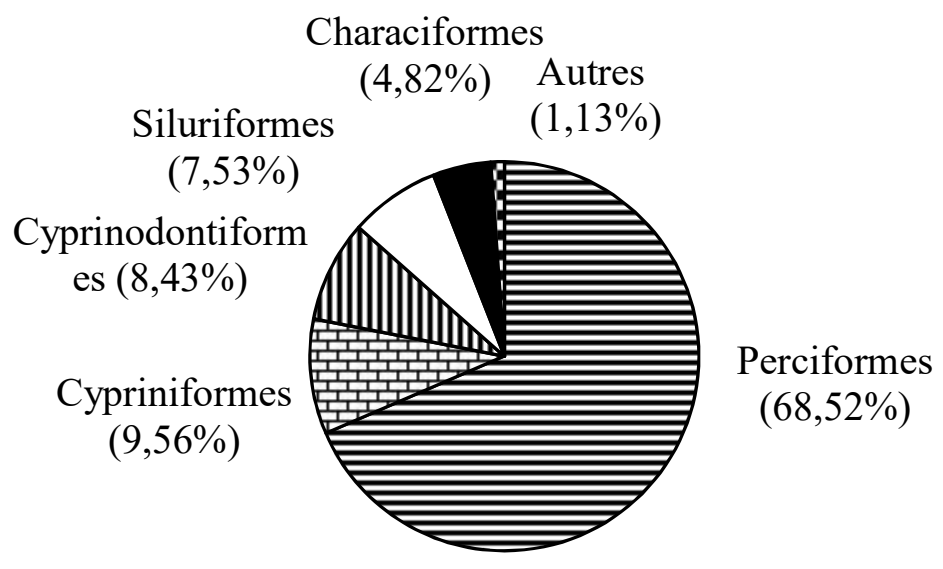

Figure 2 : Pourcentage numérique des principaux ordres de poissons échantillonnés dans la rivière Dodo.

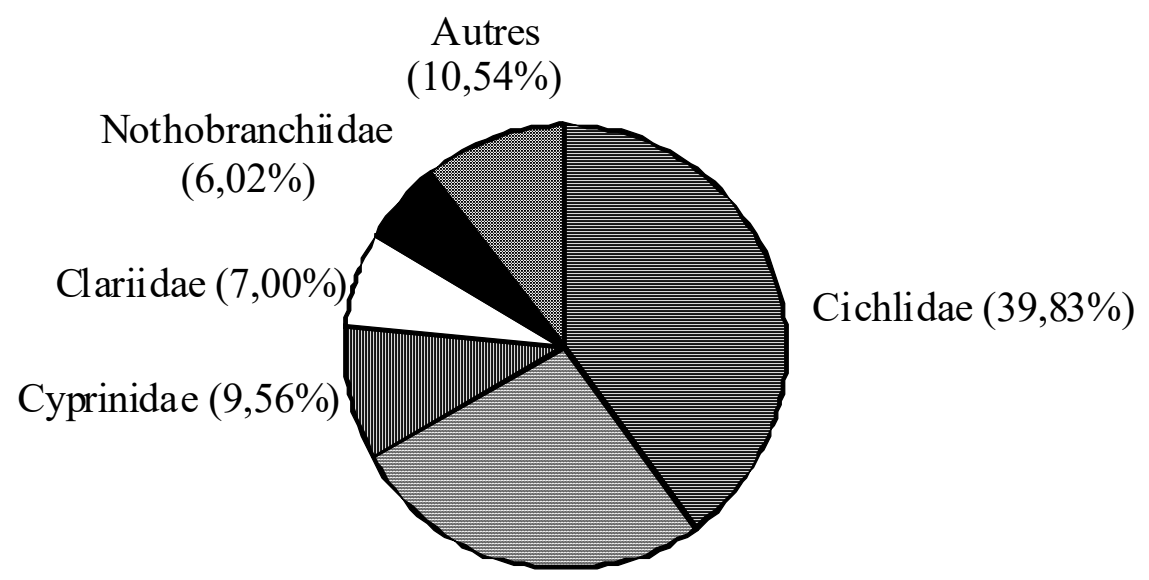

Gobiidae $(27,03 \%)$ 
Figure 3 : Pourcentage numérique des principales familles de poissons échantillonnés dans la rivière Dodo.

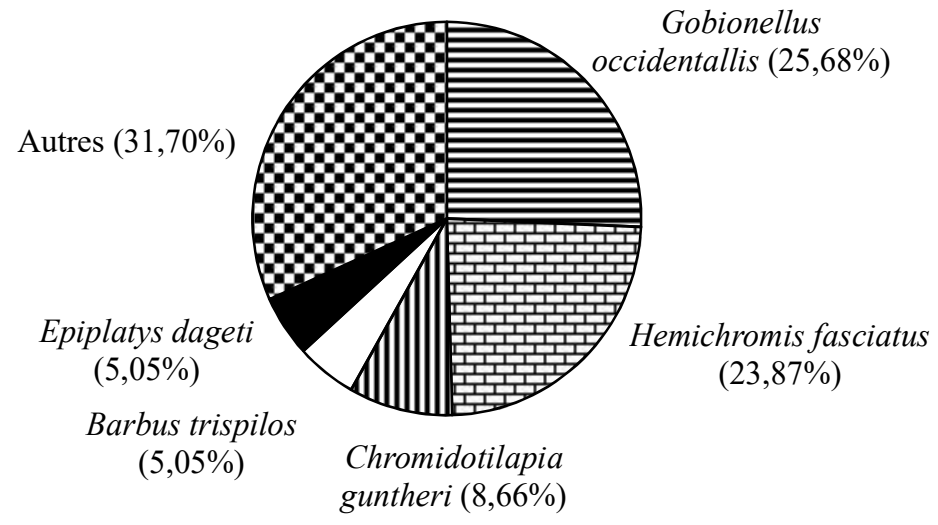

Figure 4 : Pourcentage numérique des principales espèces de poissons échantillonnés dans la rivière Dodo.

Zonation longitudinale de la rivière: Sur le cours principal et l'affluent principal, les stations les plus éloignées de l'océan Atlantique où des espèces à affinité marine ou estuarienne ont été capturées sont respectivement D13 (avec l'espèce Liza falcipinnis) et D34 (avec l'espèce Dalophis boulengeri). Ainsi, le cours supérieur comprend les stations D1 à D12 et D28 à D33. Le cours inférieur renferme les stations D13 à D27, D34 et D35 (Figure 1).

Distribution et occurrence de l'ichtyofaune: Dix espèces (Petrocephalus bovei, Chrysichthys nigrodigitatus, Amphilius atesuensis, Heterobranchus longifilis, Enneacampus kaupi, Sarotherodon melanotheron, Tilapia mariae, Tilapia zillii, Thysochromis ansorgii et Kribia nana) ont été échantillonnées seulement dans le cours supérieur de la Dodo et quatre espèces (Dalophis boulengeri, Microphis brachyurus aculeatus, Epiplatys chaperi et Gobionellus occidentalis) ont été capturées uniquement dans le cours inférieur (Tableau 1). Les autres espèces (Brycinus longipinnis, Barbus ablabes, Barbus trispilos, Clarias buettikoferi, Heterobranchus isopterus, Aplocheilichthys schioetzi, Epiplatys dageti, Parachanna obscura, Chromidotilapia guntheri, Hemichromis fasciatus, Tilapia guineensis, Tilapia hybride, Awaous lateristriga, Parasicydium bandama et Ctenopoma petherici) sont présentes aussi bien dans le cours inférieur que le cours supérieur. Les pourcentages d'occurrence calculés permettent de noter que les espèces qui ont une plus large distribution sont Chromidotilapia guntheri capturée dans 22 stations sur un total de 30 stations (soit 73,33\% d'occurrence) et Hemichromis fasciatus (20 stations ; 66,67\%) (Tableau 1). Elles sont suivies de Brycinus longipinnis et d'Epyplatys dageti (12 stations chacune ; $40 \%$ ). Cinq espèces accessoires (Barbus ablabes, Barbus trispilos, Heterobranchus isopterus, Aplocheilichthys schioetzi et Parachanna obscura), sept espèces accidentelles (Amphilius atesuensis, Clarias buettikoferi, Enneacampus kaupi, Tilapia hybride, Awaous lateristriga, Parasicydium bandama et Ctenopoma petherici) et treize espèces très rares (Dalophis boulengeri, Petrocephalus bovei, Chrysichthys nigrodigitatus, Heterobranchus longifilis, Microphis brachyurus aculeatus, Epiplatys chaperi, Sarotherodon melanotheron, Tilapia guineensis, Tilapia mariae, Tilapia zillii, Thysochromis ansorgii, Gobionellus occidentalis et Kribia nana) ont été aussi recensées.

Influence des variables environnementales sur la distribution des espèces : Le test de Monte Carlo a permis de sélectionner 7 variables qui expriment à 52,29 $\%$ les corrélations espèces-variables observées. Ces variables sont: rocher $(12,02 \%)$, plantes aquatiques $(10,02 \%)$, taux d'oxygène dissous $(7,47 \%)$, mélange sable-gravier $(6,00 \%)$, bois mort-feuilles-racines $(5,66 \%)$, taux de couverture de la canopée $(5,56 \%)$ et largeur de la rivière $(5,56 \%)$ (Figure 5$)$. 


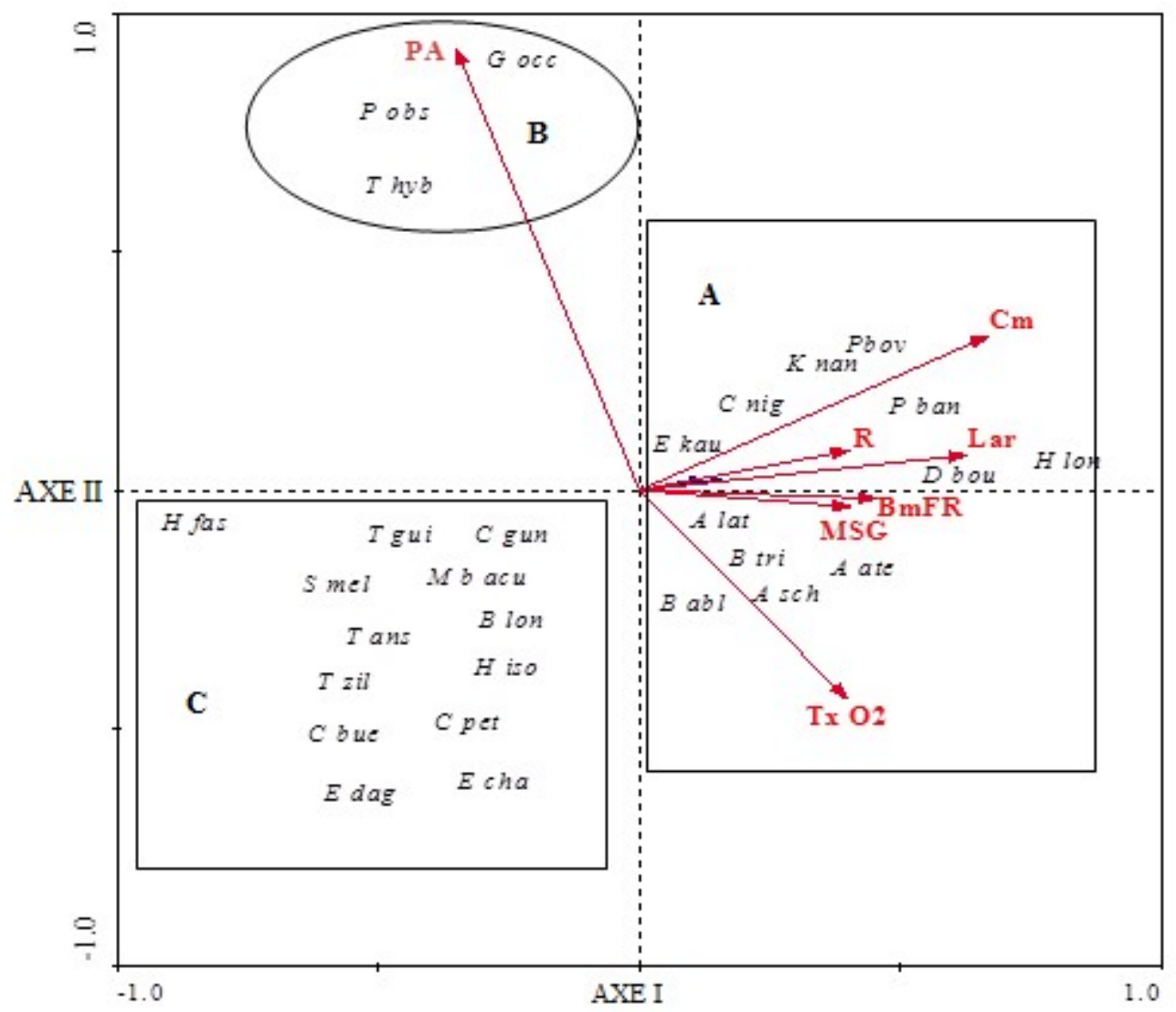

Figure 5 : Analyse canonique de correspondance (ACC) appliquée aux variables environnementales et aux espèces de poissons capturées dans la rivière Dodo. Les codes et les espèces correspondantes sont consignés dans le tableau 1. $\mathrm{PA}=$ plantes aquatiques $; \mathrm{Cm}=$ canopée moyenne $; \mathrm{R}=$ rocher $; \mathrm{Lar}=$ largeur du cours d'eau $; \mathrm{BmFR}=$ substrat de bois mort, de feuilles et de racines ; MSG = substrat de sable et de gravier ; Tx O2 = taux d'oxygène dissous.

L'influence de ces variables sur la distribution des poissons est significative $(p<0,05)$. Les axes I $(\lambda 1=0$, 193) et II $(\lambda 2=0,103)$ permettent de dégager trois groupes d'espèces : le premier groupe $(A)$ est composé des espèces Chrysichthys nigrodigitatus, Enneacampus kaupi, Kribia nana, Petrocephalus bovei, Parasicydium bandama, Dalophis boulengeri, Heterobranchus longifilis, Awaous lateristriga, Barbus trispilos, Aplocheilichthys schioetzi, Barbus ablabes et Amphilius atesuensis. Ce groupe est corrélé positivement aux variables taux de couverture de la canopée, rocher, largeur du cours d'eau, bois morts-feuilles-racines, mélange sable-gravier et taux

\section{DISCUSSION}

Teugels et al. (1988), Paugy et al. (1994) et Hugueny \& Lévêque (2006) ont respectivement signalé 21, 25 et 15 espèces de poissons dans la rivière Dodo, contre 39 espèces identifiées lors de la présente étude. Quatre ordres (Anguilliformes, Clupeiformes, Osteoglossiformes d'oxygène dissous. Le second groupe (B) comprend les espèces Gobiollenus occidentallis, Parachanna obscura et Tilapia hybride qui sont associées aux plantes aquatiques. Les espèces Hemichromis fasciatus, Heterobranchus isopterus, Clarias buettikoferi, Sarotherodon melanotheron, Tilapia zillii, Thysochromis ansorgii, Tilapia guineensis, Chromidotilapia guntheri, Epiplatys dageti, Epiplatys chaperi, Ctenopoma petherici, Brycinus longipinnis et Microphis brachyurus aculeatus qui constituent le troisième groupe (C) sont corrélées négativement aux différentes variables.

et Syngnathiformes), 9 familles (Ophichthidae, Clupeidae, Notopteridae, Mormyridae, Claroteidae, Schilbeidae, Syngnathidae, Mugilidae et Eleotridae), 22 espèces (Dalophis boulengeri, Pellonula leonensis, Papyrocranus afer, Marcusenius ussheri, Petrocephalus bovei, Brycinus 
macrolepidotus, Micralestes occidentalis, Chrysichthys nigrodigitatus, Schilbe mandibularis, Amphilius atesuensis, Clarias buettikoferi, Heterobranchus isopterus, H. longifilis, Enneacampus kaupi, Microphis brachyurus aculeatus, Aplocheilichthys schioetzi, Tilapia zillii, Thysochromis ansorgii, Liza falcipinnis, Gobionellus occidentalis, Parasicydium bandama et Kribia nana) et un Tilapia hybride sont signalés pour la première fois dans la rivière Dodo. En Côte d'Ivoire, avant la présente étude, l'espèce Parasicydium bandama était connue seulement du fleuve Bandama (Harrison \& Miller, 1992) et de la rivière Niouniourou (N'Douba et al., 2003). Selon Harrison et al. (2003), cette espèce pourrait être plus largement distribuée. Sa faible occurrence actuellement connue serait due au fait que Parasicydium bandama fuit facilement et est difficile à capturer.

Dix espèces préalablement signalées par Teugels et al. (1988) et Paugy et al. (1994) n'ont pas été échantillonnées au cours de la présente étude. Ce sont : Pellonula vorax, Brycinus derhami, Barbus parawaldroni, Micropanchax pfaffi, Poropanchax normani, Poropanchax rancureli, Aplocheilichthys spilauchen, Epiplatys olbrechtsi, Fundulopanchax walkeriet Sicydium brevifile. II est remarquable que la plupart de ces espèces soient de petite taille et des Cyprinodontiformes. En raison de leur petite taille et de l'occupation de cours d'eau peu profonds, ces espèces sont capturées plus aisément à la pêche électrique qu'avec un filet maillant. Leur absence de nos échantillons ne peut donc être imputée aux moyens de capture. Cependant, au stade actuel de nos investigations, il serait hasardeux de conclure à une disparition de ces espèces de la rivière Dodo. Toutefois, l'état de conservation de certaines d'entre elles s'avère préoccupant. Ainsi $B$. derhami est qualifiée d'espèce vulnérable (Awaïss \& Lalèyè, 2010) en raison de son aire de répartition antérieurement limitée à trois bassins (Néro, Dodo et Tabou) du sud-ouest forestier de la Côte d'Ivoire qui est potentiellement exposé au conséquences de la déforestation, de la pollution et de l'expansion de l'agriculture. Par ailleurs, de précédents échantillonnages dans la rivière Néro n'ont pas permis de capturer cette espèce. Les espèces Barbus parawaldroni, Epiplatys olbrechtsi et Fundulopanchax walkeri sont également menacées par la déforestation et l'industrie minière (Entsua-Mensah, 2010a; Entsua-Mensah, 2010b et Entsua-Mensah \& Lalèyè, 2010). L'absence du Poeciliidae Aplocheilichthys spilauchen de nos captures pourrait se justifier par le fait que cette espèce a plutôt une affinité pour les eaux saumâtres et colonise rarement les eaux douces (Wildekamp \& Van Der Zee, 2003). La relation mathématique définie par Daget et Iltis (1965) et les travaux de Hugueny (1989) ont estimé respectivement la capacité théorique de la rivière Dodo entre 26 et 27 espèces, et 18 espèces. Le nombre d'espèces recensées au cours de la présente étude ( $n=$ 39 dont 31 espèces dulçaquicoles) est supérieur à la capacité théorique déterminée par ces auteurs. Nous convenons donc avec Gourène et al. (1999) et Lalèyè et al. (2004) que la prédiction de ces auteurs n'est pas assez précise ou bien qu'elle est fondée sur d'autres résultats moins complets. L'inventaire qualitatif a révélé que les Perciformes, les Siluriformes et les Cichlidae présentent une plus grande diversité taxinomique. La dominance de ces groupes est commune à plusieurs bassins ivoiriens: Gô (Koné et al., 2003a), Boubo (Kouamélan et al., 2003), San Pedro (Koné et al., 2003b), Niouniourou (N'Douba et al., 2003), Comoé (Yao et al., 2005), Sassandra (Kouamé et al., 2008) et Bandama (Aboua et al., 2010).

Sur la base des abondances numériques et des pourcentages d'occurrence, les différentes espèces de poissons de la rivière Dodo peuvent être classées en 4 grands groupes :

(1) espèce à aire de répartition étendue et abondante: Hemichromis fasciatus ;

(2) espèces à aire de répartition étendue et peu abondantes: Brycinus longipinnis, Epiplatys dageti et Chromidotilapia guntheri ;

(3) espèces à aire de répartition réduite et abondante: Gobionellus occidentallis ;

(4) espèces rares, c'est-à-dire à aire de répartition réduite et peu abondante: Barbus ablabes, Barbus trispilos, Clarias buettikoferi, Heterobranchus isopteri, Aplocheilichthys schioetzi, Parachanna obscura, Tilapia guinensis, Tilapia hybride, Sarotherodon melanotheron, Parasicydium bandama, Dalophis boulengeri, Petrocephalus bovei, Chrysichthys nigrodigitatus, Amphilius atesuensis, Heterobrancus longifilis, Enneacampus kaupi, Microphis brachyurus aculeatus, Epiplatys chaperi, Tilapia mariae, Tilapia zillii, Thysochromis ansorgii, Awaous lateristriga, Kribia nana et Ctenopoma petherici.

En Côte d'Ivoire, le caractère ubiquiste de Hemichromis fasciatus a été aussi noté dans les bassins du Boubo (Kouamélan et al., 2003), Niouniourou (N'Douba et al., 2003), Comoé (Yao, 2006) et du Bandama (Aboua et al., 2010). La vaste distribution de cette espèce est 
également reconnue à l'échelle du continent africain (Teugels \& Thys van den Audenaerde, 2003) où sa tolérance à la pollution et aux milieux pauvres en oxygène lui permet de coloniser une diversité de biotopes (Lévêque \& Paugy, 2006b ; Mamonekene et al., 2006 ; Obasohan, 2008). Une corrélation significative a été mise en évidence entre la distribution du peuplement ichtyologique et 7 variables environnementales : oxygène dissous ; fermeture de la canopée ; substrat de bois mort, feuilles et racines; largeur du cours d'eau; substrat rocheux; plantes aquatiques; substrat de sable et de gravier. Pour les quatre premières variables, ce résultat concorde avec ceux obtenus par Kouamélan et al. (2003) et Kouadio et al. (2006) respectivement sur les rivières Boubo et Mé. Aboua et al. (2010) ont également mis en évidence le rôle significatif des variables oxygène dissous, rocher, largeur, plantes aquatiques et mélange sable-gravier sur la distribution de l'ichtyofaune dans le fleuve Bandama. II existe un lien évident entre la végétation riveraine, le taux de couverture de la canopée et le substrat de bois mort, de feuilles et de racines ; tant par la nature de ces variables que par leur rôle sur le milieu aquatique et l'ichtyofaune. Outre le développement de substrats et de débris végétaux, la canopée influence la température atmosphérique et celle de l'eau, la qualité et la quantité de la lumière qui pénètre dans l'eau,

\section{CONCLUSION}

La présente étude a permis d'actualiser les données relatives à la faune ichtyologique de la rivière Dodo. Vingt-trois espèces, dont une hybride, sont signalées pour la première fois. Dix espèces préalablement inventoriées par divers auteurs n'ont pas été capturées. l'ensoleillement, l'ombrage, la production primaire et le réseau trophique (Tabacchi et al., 2000 ; Pusey \& Arthington, 2003; Cetra \& Petrere, 2007; King \& Warburton, 2007; Beltrão et al., 2009). Les débris végétaux favorisent la prolifération de larves et d'invertébrés et constituent une source de nourriture pour certains organismes aquatiques dont les poissons. Ils peuvent servir d'habitats ou de refuges à des taxons vulnérables. Les débris végétaux agissent également sur les paramètres physico-chimiques de l'eau tels que le taux de solides dissous, la conductivité, le $\mathrm{pH}$ et la turbidité. En somme, à travers la canopée et les débris végétaux, la végétation riveraine joue un rôle primordial dans la distribution, la biologie, l'écologie, la structure, la conservation et la gestion des communautés aquatiques en général et ichtyologiques en particulier

Les macrophytes jouent également un rôle essentiel dans le développement des poissons et d'autres organismes (invertébrés, oiseaux d'eau) (Cronin et al., 2006 ; Thomaz et Cunha, 2010). Ils leur servent de nourriture, d'habitat, de refuge ou de frayère et participent à la régulation du taux d'oxygène dissous par la photosynthèse Cependant, leur prolifération constitue un problème environnemental majeur à cause de leur impact nuisible sur la navigation, la baignade, la pêche, l'agriculture et la santé publique (Trinidad et al., 2008 ; Kouamé et al., 2009).

Face à la forte anthropisation des berges et du bassin de la rivière Dodo, un suivi permanent de l'état de la faune ichtyologique s'avère nécessaire dans l'élaboration des mesures adéquates à prendre pour éviter une perte de la biodiversité.

\section{REMERCIEMENTS}

Les auteurs remercient les autorités administratives, les populations locales riveraines de la rivière Dodo et les techniciens pour leur contribution à la collecte des données.

\section{RÉFÉRENCES BIBLIOGRAPHIQUES}

Aboua BRD, N'Zi KG, Kouamelan EP, Berté S, Bamba $M, 2010$. Organisation spatiale du peuplement de poissons dans le Bandama. International Journal of Biological and Chemical Sciences 4 (5): 1480-1493.

Awaïs $A$. and Lalèyè $P, 2010$. Brycinus derhami in: IUCN 2011. IUCN Red List of Threatened Species. Version 2011.2, Downloaded on 17 May 2012. www.iucnredlist.org.
Beltrão GBM, Medeiros ESF, Ramos RTC, 2009. Effects of riparian vegetation on the structure of the marginal aquatic habitat and the associated fish assemblage in a tropical Brazilian reservoir. Biotaneotropica 9 (4): 37-43.

Brummett RE, Lazard J, Moehl J, 2008. African aquaculture: Realizing the potential. Food Policy 33: 371-385.

Cetra M. and Petrere JM, 2007. Associations between fish assemblage and riparian vegetation in the 
Corumbataí River Basin (SP). Brazalian Journal of Biological 67 (2): 191-195.

Cronin G, Lewis WM, Schiehser MA, 2006. Influence of freshwater macrophytes on the littoral ecosystem structure and function of a young Colorado reservoir. Aquatic Botany 85: 37-43.

Daget J. et Iltis A, 1965. Poissons de Côte d'Ivoire (eaux douces et saumâtres). Mémoires de l'Institut Fondamental de l'Afrique Noire (IFAN), Paris, France, $74.385 \mathrm{pp}$.

Entsua-Mensah M, 2010a. Barbus parawaldroni. In: IUCN 2011. IUCN Red List of Threatened Species. Version 2011.2, Downloaded on 17 May 2012. www.iucnredlist.org.

Entsua-Mensah M, 2010b. Epiplatys olbrechtsi ssp. olbrechtsi. In: IUCN 2011. IUCN Red List of Threatened Species. Version 2011.2, Downloaded on 17 May 2012. www.lucnredlist.org.

Entsua-Mensah M. and Lalèyè $P, 2010$. Fundulopanchax walkeri. In: IUCN 2011. IUCN Red List of Threatened Species. Version 2011.2, Downloaded on 17 May 2012, www.lucnredlist.org.

Gbenyedji JNBK, Anani KE, Amevoin K, Glitho IA, 2011. Diversité spécifique des termites (Isoptera) dans deux plantations de tecks (Tectona grandis $L$.) au sud du Togo. International Journal of Biological and Chemical Sciences 5 (2): 755765.

Gourène G, Teugels GG, Hugueny B, Thys van den Audenaerde DFE, 1999. Évaluation de la diversité ichtyologique d'un bassin ouest-africain après la construction d'un barrage. Cybium 23 (2): 147-160.

Harrison IJ et Miller PJ, 1992. Gobiidae. In: Faune des poissons d'eaux douces et saumâtres de l'Afrique de l'Ouest (C. Lévêque, D. Paugy \& G.G. Teugels (eds)). Tome 2. MRAC, Tervuren \& ORSTOM, Paris: pp 798-821.

Harrison IJ, Miller PJ, Pezold F, 2003. Gobiidae. In: Faune des poissons d'eaux douces et saumâtres de l'Afrique de l'Ouest (D. Paugy, C. Lévêque \& G.G. Teugels (eds)). Tome 2. IRD Éditions, MNHN, Paris \& MRAC, Tervuren : pp 625-666.

Hugueny $B, 1989$. Biogéographie et structure des peuplements de poissons d'eau douce de l'Afrique de l'Ouest: Approches quantitatives.
Thèse de Doctorat, Université Paris 7, France. $295 \mathrm{pp}$.

Hugueny B. et Lévêque C, 2006. Richesse en espèces des peuplements de poissons. In: Les poissons des eaux continentales africaines: diversité, écologie, utilisation par l'homme (C. Lévêque \& D. Paugy (eds)). Éditions IRD, Paris : pp 263275.

Kamdem Toham A. and Teugels GG, 1998. Diversity patterns of fish assemblages in the Lower Ntem River Basin (Cameroon), with notes on potential effect of deforestation. Archiv für Hydrobiologie 141: 421-446.

King S. and Warburton K, 2007. The environmental preferences of three species of Australian freshwater fish in relation to the effects of riparian degradation. Environmental Biology of Fishes 78: 307-316.

Koné T, Teugels GG, N'Douba V, Gooré Bi G, Kouamélan EP, 2003a. Premières données sur l'inventaire et la distribution de l'ichtyofaune d'un petit basin côtier ouest-africain : rivière Gô (Côte d'Ivoire). Cybium 27(2): 101-106.

Koné T, Teugels GG, N'Douba V, Kouamélan EP, Gooré Bi G, 2003b. Fish assemblages in relation to environmental gradients along a small west African coastal basin, the San Pedro River, Ivory Coast. African Journal of Aquatic Science 28 (2): 163-168.

Kouadio NF, Kouamélan EP, N'Douba V, Koné T, Snoeks J, Ollevier F, 2006. Update of fish biodiversity and impact of human activities of the community structure, Mé River (Ivory Coast). Journal of Biology Sciences 6: 805-814.

Kouamé KA, Yao SS, Gooré Bi G, Kouamélan EP, N'Douba V, Kouassi NJ, 2008. Influential environmental gradients and patterns of fish assemblages in a West African basin. Hydrobiologia 603: 159-169.

Kouamé KKV, Yapo OB, Tidou-Boga AS, Mambo VA, Seka A, Houenou P, 2009. Physico-chemical factors involved in rivers and lagoons invasion by water hyacinth, Côte d'Ivoire. International Journal of Biological and Chemical Sciences. 3(6): 1445-1458.

Kouamélan EP, Teugels GG, N'Douba V, Gooré Bi G, Koné $T, 2003$. Fish diversity and its relationships with environmental variable in a West African basin. Hydrobiologia 505: 139-146. 
Lalèyè $P$, Chikou A, Philippart JC, Teugels GG, Vanderwalle $P, 2004$. Etude de la diversité ichtyologique du bassin du fleuve Ouémé au Benin (Afrique de l'Ouest). Cybium 28 (4): 329339.

Lévêque C. et Paugy D, 2006a. Impacts des activités humaines. In: Les poissons des eaux continentales africaines: diversité, écologie, utilisation par l'homme. (C. Lévêque \& D. Paugy (eds)). Editions de l'IRD, Paris : pp 395-413.

Lévêque C. et Paugy D, 2006b. Peuplements des cours d'eau et des biotopes associés. In: Les poissons des eaux continentales africaines: diversité, écologie, utilisation par l'homme (C. Lévêque \& D. Paugy (eds)). Éditions de l'IRD, Paris : pp 309-320.

Mamonekene V, Lavoué S, Pauwels OSG, Mve Beh JH, Mackayah JE, Tchignoumba L, 2006. Diversité des poissons de Rabi et Gamba, province de l'Ogooué-Maritime, Gabon. Bulletin of the Biology Society of Washington 12: 65-78.

N'Douba V, Kouamélan EP, Koné T, Teugels GG, Gooré $\mathrm{Bi}$ G, 2003. Premières données sur le peuplement ichtyologique de la rivière Niouniourou : petit bassin côtier ouest-africain (Côte d'Ivoire). Bioterre, Revue Internationale des Sciences de la vie et de la terre 3 (1): 107116.

Obasohan EE, 2008. Bioaccumulation of chromium, copper, maganese, nickel and lead in a freshwater cichlid, Hemichromis fasciatus from Ogba River in Benin City, Nigeria. African Jouranal of General Agriculture 4 (3): 141-152.

Paugy D, Lévêque C, Teugels GG, 2003a. Faune des poissons d'eaux douces et saumâtres de l'Afrique de l'Ouest. Tome 1. IRD Éditions, MNHN, Paris, France \& MRAC, Tervuren, Belgique. $457 \mathrm{pp}$.

Paugy D, Lévêque C, Teugels GG, 2003b. Faune des poissons d'eaux douces et saumâtres de l'Afrique de l'Ouest. Tome 2. IRD Éditions, MNHN, Paris, France \& MRAC, Tervuren, Belgique. $815 \mathrm{pp}$.

Paugy D, Traoré K, Diouf PS, 1994. Faune ichtyologique des poissons des eaux douces d'Afrique de l'ouest. In: Diversité Biologique des poissons des eaux douces et saumâtres d'Afrique (Teugels GG, Guegan JF, Albaret J.J. (eds)).
Annales du Musée Royal d'Afrique Centrale, Tervuren : pp 35-66.

Pusey BJ. et Arthington AH, 2003. Importance of the riparian zone to the conservation and management of freshwater: a review. Marine and Freshwater Research 54: 1-16.

Tabacchi E, Lambs L, Guilloy H, Planty-Tabacchi AM, Muller E, Décamps H, 2000. Impact of riparian vegetation on hydrological processes. Hydrological Processes. 14: 2959-2976.

Ter Braak CJF. et Šmilauer P, 2002. Canoco reference manual and Canodraw for Windows user's guide: Software for Canonical Community Ordination (version 4.5). Microcomputer Power (Ithaca NY, USA). 500 pp.

Teugels GG. and Thys Van den Audernaerde DFE, 2003. Cichlidae. In: Faune des poissons d'eaux douces et saumâtres de l'Afrique de l'Ouest (D Paugy C, Lévêque C, Teugels GG (eds)). Tome 2. IRD, MNHN, Paris \& MRAC, Tervuren: $p p$ 521-600.

Teugels GG, Lévêque C, Paugy D, Traoré K, 1988. État des connaissances sur la faune ichtyologique des bassins côtiers de Côte d'Ivoire et de l'Ouest du Ghana. Revue d'Hydrobiologie Tropicale 21 (3): 221-237.

Thomaz SM and Cunha ER, 2010. The role of macrophytes in habitat structuring in aquatic ecosystems: methods of measurement, causes and consequences on animal assemblages' composition and biodiversity. Acta Limnologica Brasiliensia 22 (2): 218-236.

Trinidad RT, Elsa MRL, Gloria LG, Eva AP, Ricardo ML, Juan MSG. 2008. The Water. Aquatic Invasions 3(1): 42-53.

Wildekamp RH. et Van der Zee JR, 2003. Poeciliidae. In: Faune des poissons d'eaux douces et saumâtres de l'Afrique de l'Ouest (C. Lévêque, D. Paugy and G.G. Teugels (eds)). Tome 2. MRAC, Tervuren, MNHN \& IRD, Paris: pp 300324.

Wu J, Wang J, He Y, Cao W, 2011. Fish assemblage structure in the Chishui River, a protected tributary of the Yangtze River. Knowledge and Management of Aquatic Ecosystems 400 (11): 1-14.

Yao SS, 2006. Etude de la diversité biologique et de l'écologie alimentaire de l'ichtyofaune d'un hydrosystème ouest africain : cas du bassin de 
Kamelan et al. J. Appl. Biosci. 2013. Ichtyofaune de la rivière Dodo, Côte d'ivoire : mise à jour et influence des variables environnementales sur la distribution des espèces

la Comoé (Côte d'Ivoire). Thèse de Doctorat. Université Cocody-Abidjan, Côte d'Ivoire. 194 pp.

Yao SS, Kouamélan EP, Koné T, N'Douba V, Gooré Bi G, Ollevier F, Thys Van Den Audenaerde DFE,
2005. Fish communities along environmental gradients within the Comoé River basin, Côte d'Ivoire. African Journal of Aquatic Science 30 (2): 195-204. 